\title{
A IMPORTÂNCIA DO CONTROLE DE QUALIDADE DE PROCESSADORAS AUTOMÁTICAS*
}

\author{
Luís Alexandre Gonçalves Magalhães ${ }^{1}$, Ana Cecília Pedrosa de Azevedo ${ }^{2}$, Antonio Carlos \\ Pires Carvalho ${ }^{3}$
}

Resumo 0 controle de qualidade de processadoras automáticas é um dos aspectos mais importantes na implementação de programas de garantia de qualidade em radiodiagnóstico. Um programa piloto de garantia de qualidade para processadoras automáticas utilizando o método sensitométrico foi implementado no serviço de mamografia de um hospital universitário no Rio de Janeiro. Inicialmente foi feita uma avaliação das condições de funcionamento de todas as câmaras escuras do hospital. Foi constatado que apresentavam altos níveis de temperatura e umidade relativa ambiental e várias entradas de luz; nível de fog elevado, que tornava necessário um aumento de até $24 \%$ na mAs para compensar a perda de contraste; tempo de processamento seco-a-seco com erros de até $56 \%$; temperatura do revelador de $39^{\circ} \mathrm{C}\left(4^{\circ} \mathrm{C}\right.$ a mais do que o recomendado pelo fabricante); taxa de reposição dos produtos químicos com erros de até $417 \%$ acima do recomendado pelo fabricante; e índices de rejeição de filmes da ordem de $\mathbf{2 1 \%}$. Medidas sensitométricas feitas antes da implementação do projeto acusaram valores de base + fog de 0,24 densidade óptica, assim como velocidade e contraste fora dos limites recomendados. Os filmes apresentavam vários artefatos produzindo imagens de baixa qualidade. $O$ projeto piloto consistiu na implementação do programa de garantia de qualidade no setor de mamografia do hospital. Muitas outras iniciativas contribuíram para o sucesso do projeto, tais como: treinamento dos técnicos de mamografia, melhor manutenção do mamógrafo e, especialmente, o aumento do número médio de pacientes por dia, de 7 para 12, o que contribuiu para a estabilidade da processadora. Esta iniciativa permitiu que a taxa de reposição dos produtos químicos fosse reduzida a níveis aceitáveis. A implementação do controle de qualidade manteve os parâmetros sensitométricos sob controle. 0 maior ganho com a implantação do programa foi a melhoria da qualidade da imagem, sem artefatos, com redução significativa dos custos do serviço e, especialmente, a redução das doses nos pacientes, em virtude da diminuição dos índices de rejeição dos filmes para 7,7\%.

Unitermos: Controle de qualidade; Processadoras automáticas; Método sensitométrico.

Abstract The importance of quality control of automatic processors.

Quality control of automatic processors is an important issue in the implementation of quality assurance programs in diagnostic radiology. A pilot program of quality control for automatic processors using the sensitometric method was implemented in the Radiodiagnosis Service of a University Hospital in Rio de Janeiro, RJ, Brazil. An initial survey of the darkrooms showed that they had high temperature and humidity and several light leaks; fog levels were excessively high requiring an increase in $\mathrm{mAs}$ of up to $24 \%$ to compensate for contrast loss; dry to dry processing time was out of control with errors of up to $56 \%$; developer temperature was as high as $39^{\circ} \mathrm{C}\left(4^{\circ} \mathrm{C}\right.$ higher than the recommended by the manufacturers); replenishment rates presented values as high as $417 \%$ above manufacturer's recommendations; and rejection rates of radiographic films were of the order of $21 \%$ in the mammography service. Sensitometric measurements performed before the implementation of the quality control showed values of base + fog as high as 0.24 optical density and speed and contrast outside recommended limits. The radiographic films presented several artifacts that resulted in low quality images. The pilot program consisted of the implementation of the quality control in the mammography service. Several initiatives were taken including training of the technicians, improvement of maintenance of the processors and increase in the number of patients/day from 7 to 12 . This procedure allowed replenishment rates to be lowered to acceptable levels. The implementation of the quality control maintained chemical products stable keeping the sensitometric parameters under control. However, the most significant result of the program was better image quality, free from artifacts, a significant reduction in costs to the hospital and especially lower doses imparted to the patients due to the reduction in rejection rates to $7.7 \%$.

Key words: Quality control; Automatic processors; Sensitometric method.

\footnotetext{
* Trabalho realizado no Hospital Universitário Clementino Fraga Filho da Universidade Federal do Rio de Janeiro (UFRJ), Rio de Janeiro, RJ.

1. Mestre em Ciências pela Faculdade de Medicina da UFRJ.

2. Doutora, Física da Faculdade de Medicina da UFRJ e da Escola Nacional de Saúde Pública - Fiocruz.

3. Doutor, Professor Adjunto do Departamento de Radiologia da Faculdade de Medicina da UFRJ, Coordenador Adjunto de Pós-graduação em Radiologia da UFRJ.

Endereço para correspondência: Dra. Ana Cecília P. de Azevedo. Fiocruz - Escola Nacional de Saúde Pública-CESTEH. Rua Leopoldo Bulhões, 1480, Manguinhos. Rio de Janeiro, RJ. 21041-210. E-mail: acpa@ensp.fiocruz.br Recebido para publicação em 22/8/2002. Aceito, após revisão, em 3/9/2002
}

\section{INTRODUÇÃO}

No Brasil, a Portaria no $053 / 98$ da Secretaria de Vigilância Sanitária do Ministério da Saúde ${ }^{(\mathbf{1})}$ foi publicada em 1998. Um dos seus principais aspectos refere-se ao fato da obrigatoriedade da implantação 
de um programa de garantia de qualidade (PGQ) em toda instituição que faça uso de radiações ionizantes. Um PGQ tem três objetivos principais: melhorar a qualidade da imagem radiográfica, reduzir os custos e as doses fornecidas aos pacientes ${ }^{(2,3)}$.

Documentos oficiais $^{(4)}$ revelaram a situação problemática em que se encontra a radiologia no Brasil, com mais de $80 \%$ dos seus cerca de 90.000 equipamentos operando fora das especificações de desempenho desejáveis. Avaliações preliminares das condições de funcionamento do serviço de radiodiagnóstico de um hospital universitário foram feitas ${ }^{(\mathbf{5 , 6})}$, mostrando que a situação encontrada naquele hospital não se afasta da realidade nacional ${ }^{(7-10)}$.

Um dos aspectos mais importante da implantação do PGQ é ter as processadoras automáticas operando sob controle, do contrário todos os outros aspectos de qualidade serão prejudicados. O método mais usual de controle de processamento é o método sensitométrico aplicado ao sistema de processamento do serviço ${ }^{(11-13)}$. De acordo com dados da literatura, erros devidos a processamento incorreto podem representar $13 \%$ dos filmes rejeitados em um serviço ${ }^{(\mathbf{1 4 - 1 6 )}}$. O controle sensitométrico de processadoras automáticas indicará que medidas corretivas devem ser tomadas antes que a imagem radiográfica se deteriore. No entanto, antes de começar o controle de qualidade (CQ) das processadoras, é necessário avaliar as condições de operação das câmaras escuras, que devem estar operando com baixos níveis de fog ${ }^{(\mathbf{1 7 , 1 8})}$ e condições adequadas de higiene.

A qualidade da imagem, a reprodutibilidade de resultados e as doses fornecidas aos pacientes dependem do tempo de processamento e da preparação e temperatura corretas dos produtos químicos ${ }^{(19-21)}$. Freqüentemente, a exposição (e, conseqüentemente, a dose fornecida aos pacientes) é aumentada para compensar a temperatura e o tempo, permitindo um processamento mais rápido. Este procedimento é uma prática comum, especialmente em radiologia odontológica.

O primeiro protótipo de processadora automática foi apresentado em $1942^{(22)}$. Melhorias ocorreram em 1956, quando foi lançada a primeira processadora com transporte por rolos ${ }^{(\mathbf{2 2})}$ e tempo de proces- samento seco-a-seco reduzido a 90 segundos. Estas melhorias permitiram o emprego de novos tipos de produtos químicos, novos tipos de emulsão nos filmes e aumento da temperatura do revelador ${ }^{(\mathbf{1 1}, 23)}$. Em 1975, a primeira processadora de mesa foi construída ${ }^{(24)}$. Em 1987, a primeira processadora de alta velocidade (45 segundos) que utilizava filmes especiais foi comercializada. O processamento automático resultou num grande avanço na produtividade, padronizando as exposições e técnicas radiográficas, levando a uma redução das doses fornecidas aos pacientes.

\section{MATERIAL E METODO}

Sensitometria é a técnica que relaciona a resposta do filme com a exposição recebida. A curva sensitométrica, ou curva H\&D ${ }^{(22,25,26)}$ (Hurter e Driffield), relaciona o grau de enegrecimento do filme, ou densidade óptica (DO), com a exposição recebida pelo filme. Avalia o contraste, a velocidade (sensibilidade) e o valor de base+fog. Regiões de baixa densidade estão no "pé" da curva e representam a base+fog (DO sem exposição). Altas densidades estão no "ombro" da curva e representam a densidade máxima do filme. Todas as densidades úteis ao diagnóstico encontram-se na região linear da curva, que também é chamada de latitude do filme (toda a escala de cinza). O contraste é determinado pela porção reta da curva entre os pontos (ou degraus) correspondentes a 0,25 e 2,0 acima da base+fog. A velocidade do filme é o inverso da exposição necessária, em Röntgen, para produzir uma DO $=1,0$ acima da base+fog. Filmes rápidos necessitam menos exposição para obter a mesma DO que filmes lentos. O degrau de velocidade é o que se encontra mais próximo do valor $1,2(1,0+$ base+fog $)$.

O método sensitométrico consiste em expor um filme à luz de um sensitômetro, obter uma tira sensitométrica com 21 degraus e avaliar os parâmetros de exposição. Os resultados obtidos são inseridos num protocolo de testes que registra a temperatura do revelador, a velocidade, o contraste e o valor de base+fog. Quando são observadas variações maiores do que as preestabelecidas, ações corretivas devem ser tomadas.
Artefatos são quaisquer imagens irreais que aparecem no filme radiográfico processado $^{(\mathbf{1 1})}$. São “defeitos” nos filmes. Um dos maiores desafios na implantação de um PGQ, especialmente em mamografia, é a correta identificação e eliminação dos artefatos. As causas dos artefatos são: processamento, manuseio dos filmes, e, principalmente, sujeira na câmara escura. São classificados como positivos ou negativos, de acordo com a cor que apresentam (preto ou branco). Artefatos de manuseio podem originar-se antes ou depois da exposição e dependem da granulação do filme.

Os equipamentos utilizados neste trabalho foram: sensitômetro Victoreen (modelo 07-417), lâmpada UV Nuclear Associates (modelo 17-800), densitômetro Victoreen (modelo 07-443), cronômetro Aristo, fotômetro Lx Quantum Instruments Inc., termômetro digital Victoreen (modelo 07-402), higrômetro/termômetro (Micronta), medidor de pH (Merck), solução Kodak Hypo Test Solution HT-2.

$\mathrm{O}$ fog no filme radiográfico pode ser causado por radiação ionizante, luzes "seguras” (provenientes da luz de segurança) e luzes "inseguras". Luzes "inseguras" podem ser: entradas de luz branca ao redor da porta, dos passadores de filmes ou da processadora, cassetes defeituosos, mostradores de relógios, rachaduras nos tetos e ainda interruptores e tomadas do tipo "luminoso". O método mais eficaz para testar o nível de fog é expor um filme à luz do sensitômetro, sob condições normais de trabalho, e processá-lo nos tempos 1, 2 e 4 minutos após a exposição. Os resultados obtidos serão comparados ao filme "padrão" (0 minuto), que deve ter sido obtido anteriormente expondo um filme à luz do sensitômetro, porém com todas as luzes de segurança desligadas. Os resultados devem demonstrar que a diferença de DO entre o filme padrão e os filmes obtidos nos tempos 1, 2 e 4 minutos devem ser no máximo de 0,05 DO. Câmaras escuras que passam no teste de 4 minutos são consideradas em excelentes condições. As que passam no teste de 2 minutos são consideradas em boas condições. No entanto, se for aprovada apenas no teste de 1 minuto, deverá ser reavaliada.

A medida do $\mathrm{pH}$ é utilizada para avaliar a atividade química do revelador e fi- 
xador, sendo os valores recomendados, respectivamente, entre 10 e 11 para o revelador e entre 4 e 5 para o fixador ${ }^{(27)}$.

O teste de retenção do fixador é empregado para verificar a quantidade de tiossulfato (hypo) que ficou retida nos filmes após o processamento. Altas quantidades de hypo indicam que o filme foi mal lavado, o que, após algum tempo, causará uma aparência amarelada nas radiografias, prejudicando a qualidade da imagem. $\mathrm{O}$ limite máximo recomendado de hypo é de $0,02 \mathrm{~g} / \mathrm{m}^{2}$. Este teste é especialmente importante em mamografia, uma vez que estas são guardadas por muitos anos para fins de comparação ${ }^{(\mathbf{1 2})}$.

A temperatura ideal do revelador depende do tipo de filme, do ciclo de processamento e das recomendações do fabricante. A temperatura influi diretamente na velocidade do filme e, conseqüentemente, na exposição do paciente, no contraste radiográfico e no valor de base+fog. Consequientemente, é de extrema importância seguir atentamente as instruções dos fabricantes, mantendo a temperatura do revelador dentro dos limites de tolerância de $\pm 0,3^{\circ} \mathrm{C}$. A temperatura do fixador pode oscilar entre $29,4^{\circ} \mathrm{C}$ e $35^{\circ} \mathrm{C}$. Já a temperatura da água deve ficar entre $4,5^{\circ} \mathrm{C}$ e $29,5^{\circ} \mathrm{C}$. Temperaturas extremas podem causar problemas de fixação e de lavagem, além do aparecimento de artefatos.

A velocidade de transporte dos filmes deve ser constante e não variar mais que $\pm 3 \%$ das recomendações do fabricante ${ }^{(3)}$.

A taxa de reposição é definida como a quantidade de revelador e fixador que entra nos tanques da processadora após a inserção do filme na bandeja. Quando a processadora tem um baixo número de filmes por dia, a reposição por inundação ("flooded replenishment") ${ }^{(28)}$ é recomendada, a fim de manter a atividade dos produtos químicos constante. Este sistema consiste num temporizador acoplado à processadora, que é periodicamente ativado, por exemplo 20 segundos a cada 5 minutos, com $65 \mathrm{ml}$ por vez.

\section{RESULTADOS E DISCUSSÃO}

O hospital avaliado possui cinco câmaras escuras: uma no centro cirúrgico e quatro no serviço de radiodiagnóstico, sendo uma no setor de mamografia, uma no setor de hemodinâmica, e duas na radiologia geral, que chamaremos de geral 1 e geral 2 (G1 e G2).

Os resultados de avaliação ambiental das câmaras escuras podem ser vistos na Tabela 1. Podemos observar valores extremamente altos de temperatura ambiental e umidade relativa. Na época da avaliação havia problemas no sistema de ar condicionado. Os valores corretos de temperatura e umidade relativa do ar são de $10^{\circ} \mathrm{C}$ a $21^{\circ} \mathrm{C}$ e de $30 \%$ a $50 \%$, respectivamente. Nenhuma das câmaras avaliadas possui sistema de exaustão apropriado. A exaustão é indispensável, a fim de reduzir a contaminação ambiental causada pelos gases tóxicos que emanam dos tanques da processadora e a poeira. Os tanques destinados a estocar os produtos químicos já preparados devem ser mantidos fora da câmara escura.

Todas as câmaras escuras apresentaram problemas comuns: falta de lanternas de segurança apropriadas e limpeza deficien- te, com a presença de inúmeros objetos alheios ao processamento, o que causa acúmulo de poeira e, consequientemente, de artefatos no filme radiográfico. Recomenda-se que seja instalada uma pia, a fim de que o operador possa lavar constantemente as mãos, mantendo assim a higiene desejável. Uma luz ultravioleta foi utilizada para comprovar a inadequação da limpeza, não só nas câmaras escuras, como também nos chassis e "écrans". Várias entradas de luz branca foram detectadas nas câmaras escuras, sendo recomendada sua vedação.

O nível de fog foi avaliado em cada uma das quatro câmaras escuras com o teste de 0, 1, 2 e 4 minutos. A Figura 1 mostra o teste de fog para a câmara escura do centro cirúrgico. O nível de fog achado para cada uma das câmaras escuras, e que necessita aumento no mAs, foi de $21 \%, 24 \%$ e $10 \%$ para G1, hemodinâmica e centro cirúrgico, respectivamente, enquanto a câmara escura do G2 obteve velamento total do filme.

Tabela 1 Valores de temperatura e umidade relativa das câmaras escuras.

\begin{tabular}{|l|c|c|}
\hline \multicolumn{1}{|c|}{ Câmara escura } & Temperatura $\left(10^{\circ} \mathrm{C}\right.$ a $\left.21^{\circ} \mathrm{C}\right)$ & Umidade relativa (30\% a 50\%) \\
\hline Centro cirúrgico & 24 & 62 \\
Mamografia & 26 & 63 \\
Hemodinâmica & 26 & 61 \\
G1 & 28 & 59 \\
G2 & 25 & 57 \\
\hline
\end{tabular}

Obs: Todos os valores encontrados estavam incorretos.

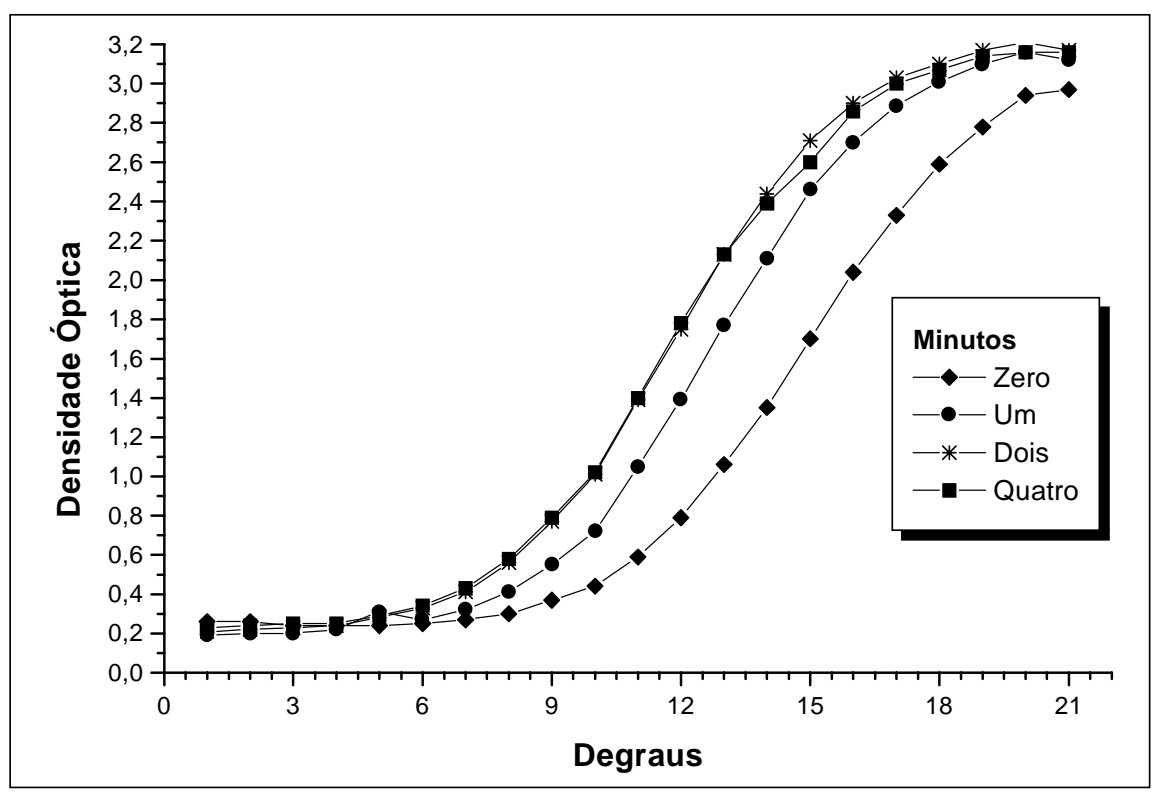

Figura 1. Curva de fog no centro cirúrgico. 
Os produtos químicos não eram preparados de acordo com as instruções dos fabricantes. Detectou-se, algumas vezes, que o pH estava incorreto, devido a diluição excessiva dos produtos químicos. Outro problema foi o repasse do fixador, o que causava perda de contraste e impossibilidade de estabilização dos parâmetros sensitométricos.

A análise da água utilizada no processamento foi feita pelo Instituto de Química da Universidade Federal do Rio de Janeiro. Os valores encontrados foram: $\mathrm{pH}$ do revelador $=5,3$ (o valor correto seria de 7). A água encontrava-se ácida, acelerando a oxidação do revelador. Foi, ainda, constatado que a dureza da água estava também incorreta (muito baixa). O resultado da análise encontra-se na Tabela 2.

Quanto ao teste de retenção de tiossulfato, todas as processadoras foram aprovadas, indicando lavagem correta dos filmes.

O resultado da avaliação do tempo de processamento seco-a-seco é apresentado na Tabela 3, sendo apenas a processadora G2 aprovada. Os erros porcentuais encontrados foram de $28 \%, 20 \%$, $56 \%$ e $22 \%$, para mamografia, G1, centro cirúrgico e hemodinâmica, respectivamente. Estes problemas são originados da falta de manutenção adequada e inexistência de manuais das processadoras. Os manuais são de extrema importância e, de acordo com a portaria no 453/98, deveriam estar disponíveis no serviço de radiodiagnóstico, pois contêm informações importantes sobre o uso e manutenção das processadoras.

Foi observado que somente no setor de mamografia a temperatura do revelador estava constante, dentro das recomendações do fabricante, enquanto nas demais processadoras encontravam-se, frequientemente, temperaturas mais altas do que o recomendado pelo fabricante. As temperaturas encontradas foram de $36,5^{\circ} \mathrm{C}$ na $\mathrm{G} 1$, $39,5^{\circ} \mathrm{C}$ na $\mathrm{G} 2,37^{\circ} \mathrm{C}$ na hemodinâmica e $33,4^{\circ} \mathrm{C}$ no centro cirúrgico. Apenas a temperatura do revelador no centro cirúrgico estava abaixo do valor recomendado pelo fabricante ${ }^{(\mathbf{2 2 , 2 3})}$. Todas as demais se encontravam acima do valor recomendado.

As taxas de reposição encontram-se nas Tabelas 4 e 5 . Uma carga de trabalho baixa da processadora requer altas taxas de reposição do revelador e do fixador, levan-

Tabela 2 Resultados da análise da água.

\begin{tabular}{|c|c|c|}
\hline Impurezas & Valores encontrados & Valores recomendados ${ }^{(\mathbf{1 1})}$ \\
\hline Sólidos dissolvidos (ppm) & 140 & 250 \\
\hline Sílica (ppm) & 10,3 & 20 \\
\hline $\mathrm{pH}$ & $5,3^{*}$ & 7 a 8,5 \\
\hline Dureza com $\mathrm{CaCO}_{3}(\mathrm{ppm})$ & $16,1^{*}$ & 40 a 150 \\
\hline Cobre, ferro, manganês (ppm) & 0,$003 ; 0,07 ; 0,01$ & 0,1 \\
\hline Cloro “livre" (ppm) & 1,5 & 2 \\
\hline Bicarbonato (ppm) & 10,4 & 150 \\
\hline Sulfato (ppm) & 6,5 & 200 \\
\hline Sulfeto (ppm) & Menos que 0,001 & 0,1 \\
\hline Cloro (ppm) & 8,6 & 25 \\
\hline
\end{tabular}

* Valores incorretos.

Tabela 3 Tempo de processamento seco-a-seco.

\begin{tabular}{|l|c|c|c|}
\hline Processadora & Tempo de processamento (s) & $\begin{array}{c}\text { Tempo de processamento } \\
\text { padrão (s) }\end{array}$ & Erro porcentual \\
\hline Mamografia & $180^{*}$ & 140 & $28 \% *$ \\
G1 & $75^{*}$ & 90 & $20 \% *$ \\
G2 & 90 & 90 & Zero \\
Centro cirúrgico & $140^{*}$ & 90 & $56 \% *$ \\
Hemodinâmica & $11^{*}$ & 90 & $22 \% *$ \\
\hline
\end{tabular}

* Valores incorretos.

Tabela 4 Taxa de reposição (sete pacientes/dia).

\begin{tabular}{|c|c|c|c|c|c|c|}
\hline Dia & $\begin{array}{c}\text { № de } \\
\text { pacientes }\end{array}$ & $\begin{array}{c}\text { Revelador } \\
\left(1.000 \mathrm{ml} / \mathrm{m}^{2}\right)\end{array}$ & $\begin{array}{c}\text { Fixador } \\
\left(1.100 \mathrm{ml} / \mathrm{m}^{2}\right)\end{array}$ & $\begin{array}{c}\text { Carga de } \\
\text { trabalho }(\mathrm{h})\end{array}$ & $\begin{array}{c}\text { Consumo de } \\
\text { revelador (\%) }\end{array}$ & $\begin{array}{c}\text { Consumo de } \\
\text { fixador (\%) }\end{array}$ \\
\hline $1^{\circ}$ & 8 & $\mathbf{1 . 5 3 2}$ & $\mathbf{1 . 5 8 3}$ & 5,6 & $\mathbf{5 3}$ & $\mathbf{4 4}$ \\
$2^{\circ}$ & 4 & $\mathbf{5 . 1 7 4}$ & $\mathbf{2 . 9 9 6}$ & 7,0 & $\mathbf{4 1 7}$ & $\mathbf{1 7 2}$ \\
$3^{\circ}$ & 6 & $\mathbf{2 . 0 5 8}$ & $\mathbf{1 . 5 7 5}$ & 7,0 & $\mathbf{1 0 6}$ & $\mathbf{4 3}$ \\
$4^{\circ}$ & 8 & $\mathbf{2 . 0 7 1}$ & $\mathbf{1 . 4 9 2}$ & 5,8 & $\mathbf{1 0 7}$ & $\mathbf{3 6}$ \\
$5^{\circ}$ & 7 & $\mathbf{2 . 7 9 4}$ & $\mathbf{1 . 8 3 6}$ & 6,5 & $\mathbf{1 7 9}$ & $\mathbf{6 7}$ \\
$6^{\circ}$ & 9 & $\mathbf{1 . 8 0 0}$ & $\mathbf{1 . 3 6 3}$ & 5,8 & $\mathbf{8 0}$ & $\mathbf{2 4}$ \\
$7^{\circ}$ & 5 & $\mathbf{3 . 1 9 4}$ & $\mathbf{2 . 1 2 9}$ & 5,9 & $\mathbf{2 1 9}$ & $\mathbf{9 4}$ \\
\hline
\end{tabular}

Obs.: Todos os valores em negrito estavam incorretos.

Tabela 5 Taxa de reposição (12 pacientes/dia).

\begin{tabular}{|c|c|c|c|c|c|c|}
\hline Dia & $\begin{array}{c}N^{\circ} \text { de } \\
\text { pacientes }\end{array}$ & $\begin{array}{c}\text { Revelador } \\
\left(1.000 \mathrm{ml} / \mathrm{m}^{2}\right)\end{array}$ & $\begin{array}{c}\text { Fixador } \\
\left(1.100 \mathrm{ml} / \mathrm{m}^{2}\right)\end{array}$ & $\begin{array}{c}\text { Carga de } \\
\text { trabalho }(\mathrm{h})\end{array}$ & $\begin{array}{c}\text { Consumo de } \\
\text { revelador (\%) }\end{array}$ & $\begin{array}{c}\text { Consumo de } \\
\text { fixador (\%) }\end{array}$ \\
\hline $1^{\circ}$ & 15 & $\mathbf{1 . 3 0 0}$ & $\mathbf{1 . 2 2 1}$ & 8,0 & $\mathbf{3 0}$ & $\mathbf{1 1}$ \\
$2^{\circ}$ & 12 & $\mathbf{1 . 4 4 4}$ & $\mathbf{1 . 2 9 7}$ & 7,0 & $\mathbf{4 4}$ & $\mathbf{1 8}$ \\
$3^{\circ}$ & 11 & $\mathbf{1 . 4 4 8}$ & 1.193 & 8,0 & $\mathbf{4 5}$ & 1 \\
$4^{\circ}$ & 13 & $\mathbf{1 . 5 0 2}$ & $\mathbf{1 . 2 0 2}$ & 8,0 & $\mathbf{5 0}$ & $\mathbf{9}$ \\
$5^{\circ}$ & 11 & $\mathbf{1 . 4 5 9}$ & 1.068 & 7,0 & $\mathbf{4 6}$ & $-3^{*}$ \\
$6^{\circ}$ & 10 & $\mathbf{1 . 3 5 5}$ & $\mathbf{1 . 2 1 9 , 5}$ & 7,0 & $\mathbf{3 5}$ & $\mathbf{1 1}$ \\
$7^{\circ}$ & 11 & $\mathbf{1 . 4 4 3}$ & 1.198 & 8,0 & $\mathbf{4 4}$ & 1 \\
\hline
\end{tabular}

Valores em negrito: consumo acima do recomendado. * Consumo do fixador abaixo do recomendado.

do, assim, à dificuldade de manter os parâmetros sensitométricos controlados. $\mathrm{O}$ número mínimo recomendado de pacientes/dia num serviço de mamografia é de, pelo menos, 15. Em um dos dias observados o número de pacientes foi de quatro, sendo detectado aumento no consumo de fixador e revelador de $417 \%$ e $172 \%$, respectivamente, acima dos valores recomendados pelo fabricante. Conseqüentemente, um filme de mama $18 \mathrm{~cm} \times 24 \mathrm{~cm}$ consumia 223,5 $\mathrm{ml}$ de revelador e $129,4 \mathrm{ml}$ de 
fixador. Estes altos valores causam redução no contraste radiográfico, aparecimento de artefatos e aumento do nível de fog. Os custos associados ao consumo dos produtos químicos também eram excessivamente altos, pois o consumo dos produtos químicos era muito mais alto que o recomendado. Aumentando o número de pacientes para $12 /$ dia, as taxas de reposição foram reduzidas e o mesmo filme $18 \mathrm{~cm} \times 24 \mathrm{~cm}$ passou a consumir apenas $56,2 \mathrm{ml}$ de revelador e 46,2 $\mathrm{ml}$ de fixador, resultando numa grande economia para o hospital.

Outro ponto muito importante é a maneira como o filme é inserido na bandeja da processadora $^{(\mathbf{1 1 , 1 2})}$. Observou-se que os técnicos não seguiam as recomendações do fabricante, posicionando seguidamente os filmes na direção horizontal em vez da vertical, a fim de possibilitar um processamento mais rápido.

Alguns exemplos de artefatos causados pela manutenção incorreta das processadoras são mostrados na Figura 2. Após a im- plementação do CQ no setor de mamografia, o aparecimento de artefatos foi reduzido, possibilitando obtenção de imagens de melhor qualidade, com mais detalhes anatômicos e níveis adequados de densidade e contraste.

As Figuras 3, 4 e 5 apresentam gráficos com os valores de base+fog, velocidade e contraste antes e depois da implementação do CQ. Deve-se observar que, antes da implantação do CQ, os limites são menos restritivos, permitindo valores de base+fog de $+0,05$ e velocidade e contraste de $\pm 0,15$. Após a implantação do CQ, aplicando-se limites mais restritivos, têm-se base+fog de $+0,03$ e velocidade e contraste de $\pm 0,10$.

Pode ser visto que antes da implantação do CQ a base+fog era de 0,24 DO e depois da implantação do CQ manteve-se em 0,20 DO (mais baixa que o valor máximo permitido de 0,21 DO). Com relação aos valores de velocidade e contraste, é apresentado, nas Figuras 3, 4 e 5, o protocolo de controle para um período de 33 dias. An- tes do CQ, estes dois parâmetros estavam fora dos limites recomendados, mesmo considerando-se valores menos restritivos de $\pm 0,15$. Após a implantação do CQ, quase todos os pontos mantiveram-se dentro de limites aceitáveis, com a processadora ficando estável e a qualidade da imagem melhorando significativamente. Apenas um ponto do contraste ficou fora dos limites preestabelecidos e foi constatado que naquele dia o fixador tinha sido repassado. O método sensitométrico imediatamente identificou o problema, que pôde ser prontamente corrigido antes que a imagem radiográfica se deteriorasse.

Com a implantação do CQ, o índice de rejeição reduziu-se de $21 \%$ para $7,7 \%$. Estes resultados estão exemplificados na Tabela 6. Esta redução deveu-se a diversas iniciativas, tais como manutenção periódica do mamógrafo, curso de treinamento para os técnicos e, especialmente, a manutenção dos parâmetros sensitométricos do processamento.

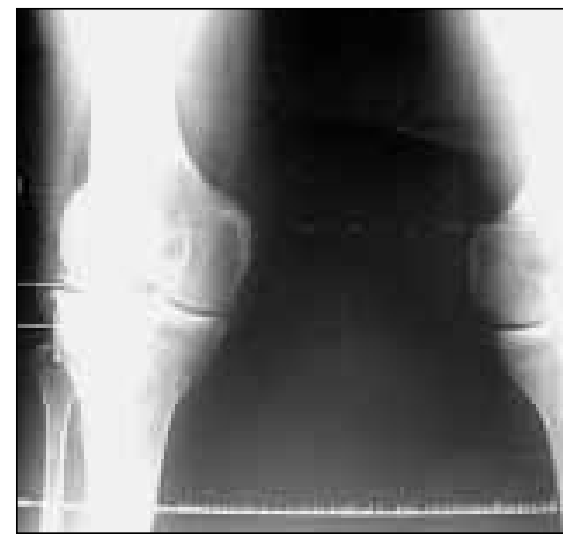

A

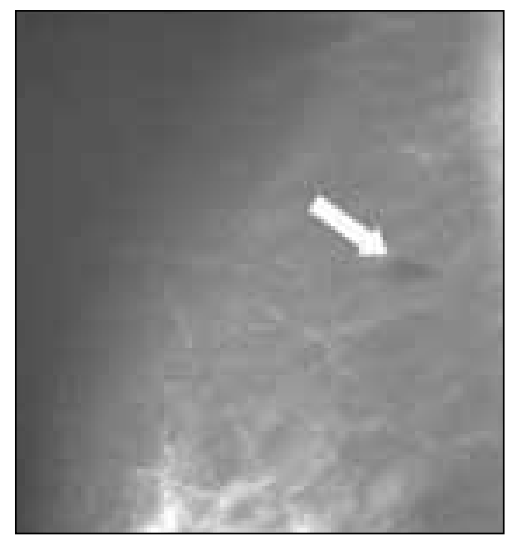

D

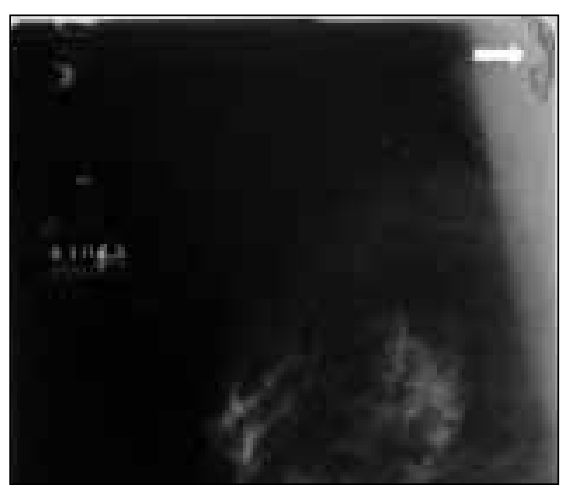

B

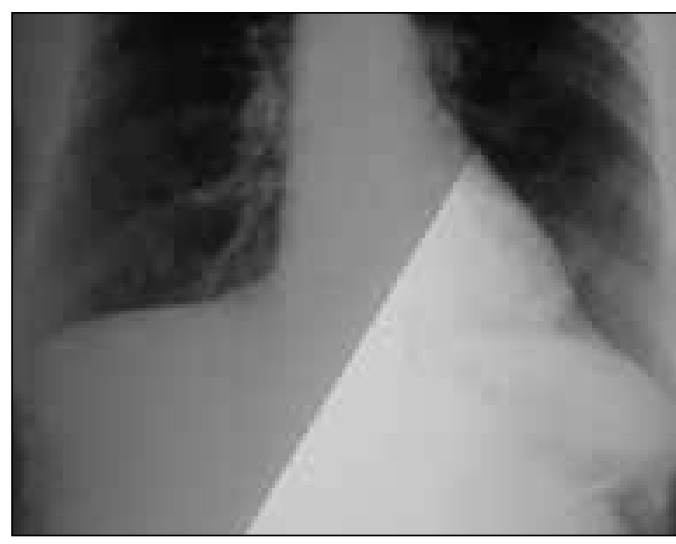

E

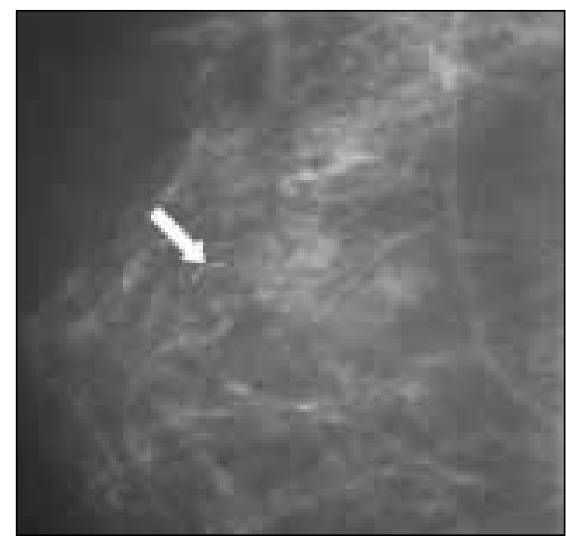

C

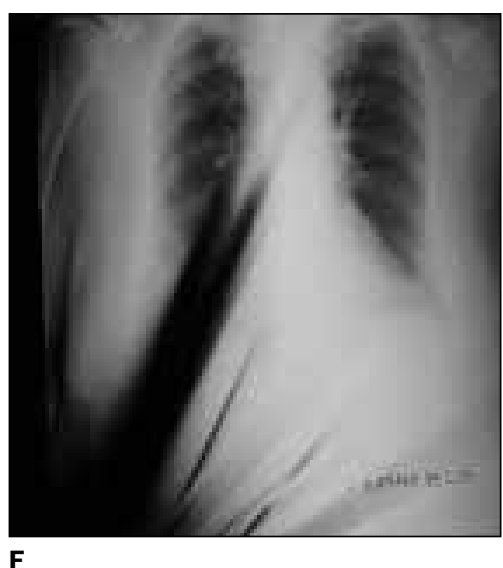

Figura 2. Exemplos de radiografias com artefatos demonstrando problemas no processamento. A: Pressão excessiva nos rolos; B: Limpeza deficiente; C: Marcas nos rolos; D: Manchas nos “écrans”; E: Filmes superpostos; F: Entradas de luz nos chassis e temperatura elevada do revelador. 


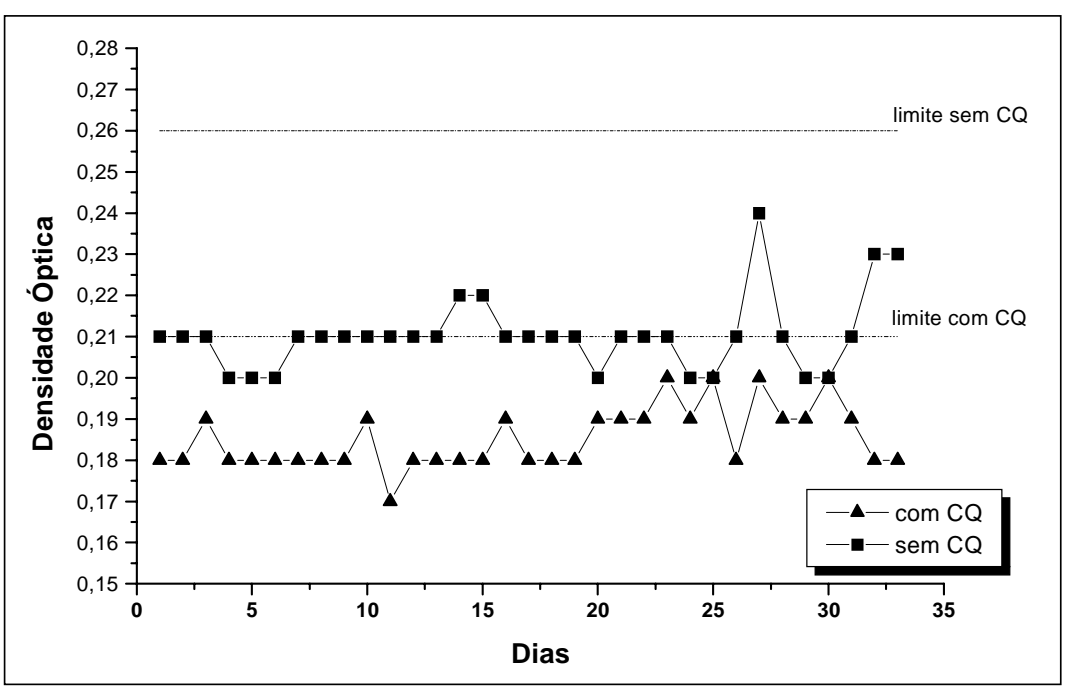

Figura 3. Base+fog antes e após a implantação do CQ.

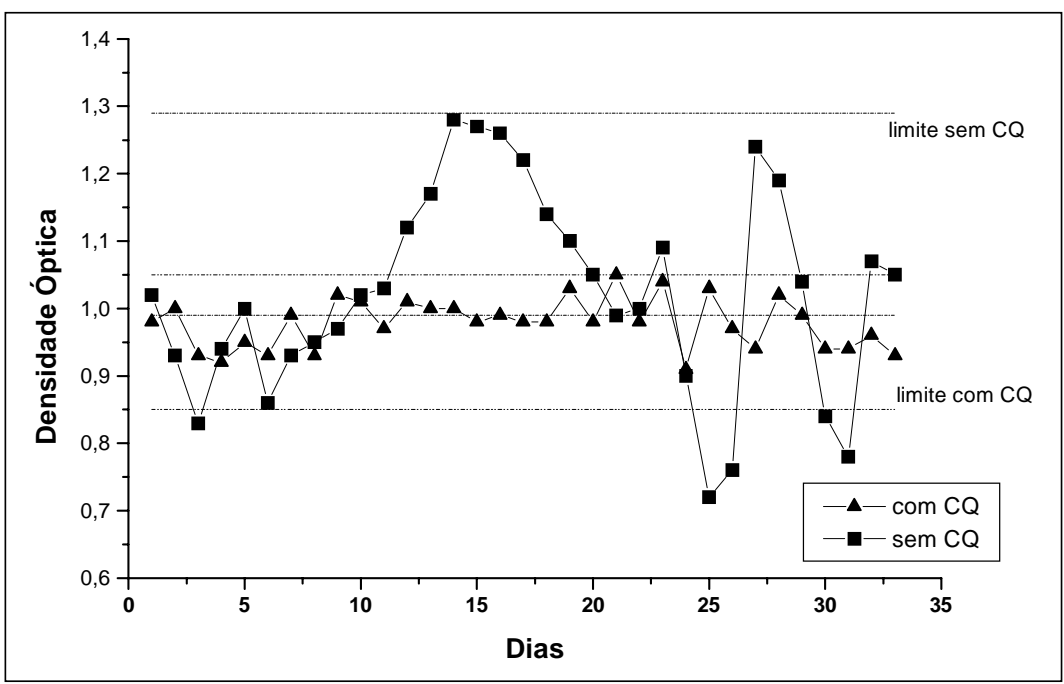

Figura 4. Velocidade antes e após a implantação do CQ.

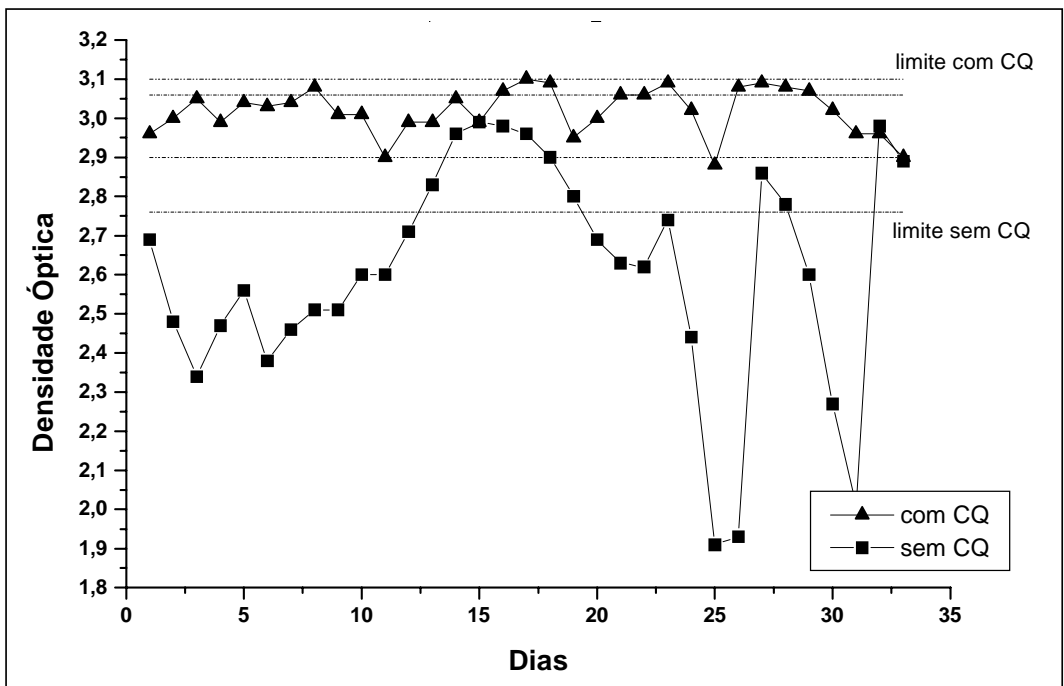

Figura 5. Contraste antes e após a implantação do CQ.
No hospital foram constatados vários problemas nas processadoras e nas câmaras escuras: rolos danificados causando artefatos nos filmes; mostradores de temperatura danificados; no G1 a bandeja da processadora estava danificada e os filmes só podiam ser inseridos para processamento do lado direito da bandeja; algumas bandejas estavam enferrujadas, causando artefatos nos filmes; as tampas das processadoras não eram mantidas abertas durante a noite, causando condensação de vapores e, conseqüentemente, contaminação dos produtos químicos; os tanques das processadoras estavam com limpeza deficiente e resíduos aderidos às paredes e aos rolos; ocasionalmente, os químicos eram preparados por longos períodos, causando oxidação e precipitação nos tanques; a taxa de reposição estava descontrolada, causando consumo excessivo de químicos; os filtros de água não eram trocados regularmente; a processadora G2 encontrava-se seguidamente com defeito e alguns dos testes não puderam ser feitos nesta processadora.

\section{CONCLUSÕES}

O programa piloto de CQ utilizando o método sensitométrico foi implementado no serviço de mamografia e incluiu: limpeza periódica da processadora, abertura da tampa superior durante a noite, preparo e manutenção correta dos produtos químicos e uma melhor manutenção da processadora.

O desempenho das processadoras e das câmaras escuras foi avaliado, a fim de possibilitar a implementação do CQ em todas as processadoras do hospital. No entanto, devido a problemas operacionais, o programa só pôde ser implantado no setor de mamografia.

O programa piloto demonstrou ser eficaz, estabilizando a processadora, melhorando a qualidade da imagem radiográfica, reduzindo o índice de rejeição de filmes e, conseqüentemente, a dose fornecida aos pacientes. Todos estes fatores foram decisivos para a redução dos custos para o hospital.

\section{Agradecimentos}

Os autores agradecem o apoio financeiro da Faperj, CNPq e Capes. 
Tabela 6 Índice de rejeição antes (setembro a novembro de 1988) e depois (março a maio de 2001) da implantação do CQ na processadora da mamografia.

\begin{tabular}{|l|c|c|c|c|}
\hline \multicolumn{1}{|c|}{ Mês } & Filmes rejeitados & Filmes aceitos & № total de filmes & Índice de rejeição \\
\hline Setembro/1998 & 47 & 133 & 180 & $26 \%$ \\
Outubro/1998 & 37 & 267 & 304 & $12 \%$ \\
Novembro/1998 & 104 & 303 & 407 & $25 \%$ \\
Março/2001 & 88 & 1.149 & 1.237 & $7 \%$ \\
Abril/2001 & 92 & 676 & 768 & $12 \%$ \\
Maio/2001 & 35 & 869 & 904 & $4 \%$ \\
\hline
\end{tabular}

\section{REFERÊNCIAS}

1. Secretaria de Vigilância Sanitária, Ministério da Saúde. Regulamento técnico. Diretrizes de proteção radiológica em radiodiagnóstico médico e odontológico. Brasília, Portaria no 453, 2/6/98.

2. National Council of Radiation Protection and Measurements. Quality assurance for diagnostic imaging equipment. Report № 99. Bethesda: NCRP, 1988:44-60.

3. Papp J. Quality management in the imaging sciences. St. Louis: Mosby, 1998.

4. Programa de avaliação de proteção radiológica em radiodiagnóstico médico. Rio de Janeiro: RXD, IRD/CNEN, 2000.

5. Azevedo ACP, Yacovenco A, Gonçalves OD, Koch HA, Tonomura ET. Avaliação do funcionamento do Serviço de Radiodiagnóstico do Hospital Universitário Clementino Fraga Filho - Universidade Federal do Rio de Janeiro. Radiol Bras 1999;32: 309-14.

6. Almeida M, Guedes G, Magalhães LAG. Levantamento do atendimento no serviço de radiologia do HUCFF. X Jornada de Iniciação Científica da UFRJ, Rio de Janeiro, novembro de 1998.

7. Corte RE. Implantação de um programa de controle de qualidade de imagens radiográficas em um hos pital de grande porte. [Dissertação de mestrado]. Ribeirão Preto: FFCLRP/USP, 2000.

8. Hourdakis CJ, Delakis J, Kamenopoulou V, Balougias H, Papageorgiou E. A pilot study on the quality control of film processing in medical radiology laboratories in Greece. Eur J Radiol 2000;33:2431.

9. Medeiros RB, Curci KA, Carrieri FCD. Controle de qualidade no processamento de imagens radiológicas. Radiol Bras 1992;25:19-21.

10. Azevedo ACP, Gonçalves OD, Magalhães LAG, Silva MO, Oliveira SR. Quality control of viewing boxes at the university hospital of the Universidade Federal do Rio de Janeiro. Physica Medica 2001; 17:89-92.

11. Magalhães LAG. Controle de qualidade de processadoras automáticas. [Dissertação de mestrado]. Rio de Janeiro: UFRJ, 2001.

12. Haus AG, Jaskulski SM. Film processing in medical imaging. Madison: Medical Physics Publishing, 1997.

13. Kofler JM Jr, Gray JE. Sensitometric responses of selected medical radiographic films. Radiology 1991;181:879-83.

14. Lymberis C, Efstathopoulos EP, Manetou A, Poudridis G. Automatic film processors' quality control test in Greek military hospitals. Eur J Radiol 1993;16:246-9.

15. McCauley B, McKinley A. Spoilt films in X-ray departments. Br J Radiol 1977;50:233-4.

16. Suleiman OH, Conway BJ, Reuter FG, Slayton RJ Automatic film processing: analysis of 9 years of observations. Radiology 1992;185:25-8.

17. Darko EO, Charles DF. Safety assessment and quality control of medical x-ray facilities in some hospitals in Ghana. Radiation Protection Board, Ghana. Atomic Energy Commission. Technical Report, 1997.

18. Gray JE. Light fog on radiographic films: how to measure it properly. Radiology 1975;115:225-7.

19. Suleiman OH, Showalter CK, Gross RE, Bunge RE. Radiographic film fog in the darkroom. Radiology 1984;151:237-8.

20. Kimme-Smith C, Rothschild PA, Bassett LW, Gold RH, Moler C. Mammographic film-processor temperature, development time, and chemistry: effect on dose, contrast and noise. AJR 1989;152:35-40.

21. Suleiman OH, Slayton RJ, Conway BJ, Reuter FG. Effects of temperature, chemistry and immersion time on x-ray film. FDA internal publication, USA.

22. Kodak. X-Omat processor's documentation.

23. Tabar L, Haus AG. Processing of mammographic films: technical and chemical considerations. Radiology 1989;173:65-9.

24. Agfa. Mammoray MR processor's documentation.

25. Bushong S. Radiologic science for technologists. St. Louis: Mosby-Year Book, 1993.

26. Christensen EE. Introduction to the physics of diagnostic radiology. 3rd ed. Philadelphia: Lea \& Febiger, 1984.

27. Stears JG, Gray JE, Winkler NT. Evaluation of $\mathrm{pH}$ monitoring as a method of processor control. Radiol Technol 1979;50:657-63.

28. Frank ED, Gray JE, Wilken DA. Flood replenishment: a new method of processor control. Radiol Technol 1980;52:271-5. 